\title{
Update of Postoperative Pain Management following Pectus Excavatum Repair
}

\author{
Jagroop Mavi* and Senthilkumar Sadhasivam \\ Department of Anesthesia, Cincinnati Children's Hospital Medical Center, Cincinnati, OH, USA
}

Received: November 22, 2013; Accepted: March 28, 2014, Published: March 31, 2014

*Corresponding author: Jagroop Mavi, Department of Anesthesia and Pain Management, Cincinnati Children's Hospital Medical Center, 3333 Burnet Avenue, MLC 2001, Cincinnati, OH 45229, USA; Tel: 513- 348-6662; Fax: 513-636-7337; Email: jagroop.mavi@cchmc.org

\begin{abstract}
Pectus deformities, mainly pectusexcavatum, are the most common chest wall deformity caused by defective growth of the sternum and surrounding costal cartilages. The technique for surgical repair is the Nuss procedure, a minimally invasive technique in which rigid metal bars are placed transthoracically beneath the sternum for a period of time until permanent remodeling of the chest wall has occurred. Postoperative pain is considerable, making multimodal analgesia the hallmark of postoperative pain control.

A thoracic epidural is the preferred analgesic technique for pectusexcavatum repair at Cincinnati Children's Hospital Medical Center (CCHMC) due to its superior pain control and minimal side effect profile. Intermitted IV narcotics or a PCA are also offered to supplement the epidural analgesia, and as the patientbegins to tolerate oral intake, they are transitioned from IV to oral pain medications. Other medications such as Methocarbamol and Diazepam as utilized as adjunct medications for relief of muscle spasms.

A retrospective study was done at CCHMC in 2012 to more clearly assess the efficacy, safety, and patient outcomes of epidurals following pectusexcavatum repair. This study yielded positive information about our experience with the pectus postoperative pain management protocol devised at this institution. CCHMC then compared their epidural analgesia outcomes with published data from Kansas.
\end{abstract}

Pectus deformitiesarethe mostcommon chestwall deformities seen in the pediatric population, occurring in approximately 1 in 300 births [1]. The deformity is caused by defective growth of the sternum and surrounding cartilage. The Nuss procedure is the technique of choice for surgical correction, and involves placing a convex bar under the sternum without resection or injury to the costal cartilages. Although classified as a minimally invasive technique, patients experience considerable postoperative pain [2]. Multimodal analgesia is the hallmark of postoperative pain control and many methods have been suggested including both regional anesthesia and patient controlled analgesia (PCA).

The length of postoperative hospitalization after pectusexcavatum repair procedures is determined by the ability to adequately control pain. The available literature suggests that thoracic epidural analgesia is the most effective means for attenuating the pain during the first few postoperative days [3]. However,a recent comparison between epidurals and IV PCA documented substantial recovery and financial advantages with PCA [4]. Surprisingly, this study had high rates of failure in successfully placing epidural catheters and keeping it beyond the first 24 postoperative hours.

A thoracic epidural is the preferred analgesic technique for pectusexcavatum repair procedures at Cincinnati Children's Hospital and Medical Center (CCHMC) due to its superior pain control and minimal side effect profile including a cognitive clear and interacting patient. Intermittent IV narcotics are available to the patient and some patients, especially in the older age groups, require the addition of a PCA to supplement the epidural analgesia. Other supplemental medications include methocarbamol 15mg/ kg every 8 hours, diazepam $0.05 \mathrm{mg} / \mathrm{kg}$ for muscle spasms and anxiety every 4 hours as needed, ketorolac $0.5 \mathrm{mg} / \mathrm{kg}$ every 6 hours for its anti-inflammatory effects, and IV acetaminophen (Tylenol) every 6 hours. As the patient begins to tolerate oral intake, they are transitioned from IV to oral pain medications including long acting oxycodone and regular Oxycodone. The epidural is often removed on the third postoperative day, which leads to a slight increase in overall pain score and even with this supplementation of medications, the transition from epidural to oral analgesics can be problematic. Intravenous narcotics are available throughout the postoperative recovery process to treat any breakthrough pain. Medication doses and frequency are adjusted as needed to provide optimal patient comfort, and they are continued for 2 weeks postoperatively; older patients sometimes need longer coverage. Refer to Table 1 for the complete protocol followed by the pain service at CCHMC.

In 2012 a retrospective study was done at CCHMC to more clearly assess the efficacy, safety and patient outcomes of epidurals following pectusexcavatum repair. The efficacy outcome measures included postoperative pain scores, success rate of epidural analgesia, need for supplemental pain medication, times to first oral intake, ambulation, and discharge. The safety outcome measures included serious side effects such as respiratory depression, neurological complications, and common opioid side effects such as nausea and itching. 
Out of 122 patients, 89 had complete data regarding postoperative pain scores, side effects, and rescue analgesics. Of these patients, $3.37 \%$ had epidurals removed on postoperative day (POD) 0 due to poor analgesia and the remaining $96.6 \%$ of patients had successful epidural analgesia. Supplemental IV PCA opioid was used in 17/89 (19\%) patients. $76 \%$ of patients had pain scores between 0 and 4 on POD 0 . A slight elevation in pain score was noted on POD 3 when the epidural was stopped. One patient, who needed supplemental PCA, developed respiratory depression. The most common side effect was nausea and vomiting, which occurred in $18 \%$ of patients. Horner's syndrome was observed $4.5 \%$ of patients. CCHMC then compared their epidural analgesia outcomes with published data from Kansas. ${ }^{3}$ The results of this comparison can be found in Table 2 .

The severity of the pectusexcavatum, as indicated by the Haller Index, was greater in the Cincinnati pectusexcavatumpatients leading to a longer surgical time compared to Kansas. The time taken to place the epidural was shorter in Cincinnati (an average of 15.54 minutes compared to95 minutes in Kansas) and the success rate was $96.5 \%$ in Cincinnati compared to $78 \%$ in Kansas with a lower percentage of patients requiring a supplemental PCA (14\% compared to 35\%). Postoperative pain scores from POD1 to 4 were slightly higher in the Kansas patients but the overall postoperative length of stay was similar at 4-5 days at both institutions.

Other forms of regional anesthesia have been described in lieu of placing a thoracic epidural and have been successful in

Table 1: Cincinnati Children's Hospital pectus excavatum postoperative pain protocol.

Epidural placement (T5-7) by anesthesia/pain team

Epidural solution: ropivacaine, $0.15 \%-0.2 \%$ with clonidine $1 \mathrm{mcg} / \mathrm{mL}$

IV acetaminophen $15 \mathrm{mg} / \mathrm{kg}$ IV every 6 hours ATC 3 days

IV methocarbamol (Robaxin) $15 \mathrm{mg} / \mathrm{kg}$ IV every 8 hours ATC 3 days

IV diazepam (Valium) $0.05 \mathrm{mg} / \mathrm{kg}$ IV every 4-6 hours as needed for

muscle spasm

IV opioids prn or PCA

Postoperative Day 1 (POD\#1)

Continue epidural analgesia, IV acetaminophen, IV methocarbamol, IV diazepam prn

Start IV ketorolac (Toradol) $0.5 \mathrm{mg} / \mathrm{kg}$ up to $15 \mathrm{mg}$ IV every 6 hours ATC

Start oxycontin 10 or $20 \mathrm{mg}$ PO every 12 hours

Postoperative Day 2 (POD\#2)

Start oxycodone, $0.1-0.2 \mathrm{mg} / \mathrm{kg}$ PO every 4 hours; schedule as ATC

Continue epidural analgesia, IV acetaminophen, IV/PO methocarbamol,

IV/PO diazepam as needed

Postoperative Day 3 (POD\#3)

Stop epidural at $6 \mathrm{AM}$ and remove on morning pain rounds

Discontinue urinary catheter 4 hours after stopping epidural

Once epidural is out: continue oxycontin/oxycodone, PO

acetaminophen every 6 hours (maximum, $75 \mathrm{mg} / \mathrm{kg} / \mathrm{d}$ ), PO

methocarbamol ATC and/or PO diazepam as needed

Start PO ibuprofen ( $10 \mathrm{mg} / \mathrm{kg} /$ dose, up to 3 times per day)

Postoperative Day 4(POD\#4)

Keep on service and help manage post-epidural pain control

If $>17$ years, history of chronic pain, or prolonged need for oral

narcotics $>2$ weeks, have patient follow up in chronic pain clinic to

assist in titration off of narcotics
Table 2:

\begin{tabular}{|c|c|c|}
\hline Patient Data and Outcome variables & Cincinnati & Kansas * \\
\hline Age (years) & $15.8+/-4.49$ & $15.5+/-2.9$ \\
\hline Haller Index & $5.57+/-3.27$ & $3.8+/-0.8$ \\
\hline Epidural Time & $15.54+/-8.97$ & $95+/-21$ \\
\hline Surgical Time & $125.61+/ 47.12$ & $53+/-18$ \\
\hline Pain Score POD 0 & $3.35+/-2.13$ & $\sim 4$ \\
\hline Pain Score POD 1 & $3.3+/-1.89$ & $\sim 3.5-4$ \\
\hline Pain Score POD 2 & $2.92+/-1.9$ & $\sim 3.5-4$ \\
\hline Pain Score POD 3 & $3.83+/-2.23$ & $\sim 4$ \\
\hline Pain Score POD 4 & $3.3+/-2.35$ & $\sim 4$ \\
\hline Epidural Success Rate & $96.50 \%$ & $78 \%$ \\
\hline Epidurals needing supplemental PCA & $14 \%$ & $35 \%$ \\
\hline Postoperative Length of stay (POD) & $4.59+/-0.91$ & $4.54+/-1.01$ \\
\hline
\end{tabular}

* Epidural vs patient-controlled analgesia for postoperative pain after pectus excavatum repair: a prospective, randomized trial [4].

* Is epidural anesthesia truly the best pain management strategy after minimally invasive pectus excavatum repair? [3]

treating postoperative pain. These include bilateral paravertebral catheters, bilateral intercostal nerve blocks, and wound catheter infusions placed by the surgeon. The use of continuous paravertebral nerve blocks for controlling postoperative pain in thoracotomy and pectusexcavatum procedures have been shown to have equal efficacy to epidural nerve blocks with a better side effect profile and lower incidence of pulmonary complications $[4,5]$. The nerves to the sternum originate from the roots of T1-T6. After exiting the lateral spinous foramina, the nerves travel in the space between the internal and innermost intercostal muscles along with the intercostal artery and vein. Anterior intercostal blocks are therefore very useful for analgesia after pectusexcavatum repair. These can be performed under ultrasound guidance with the patient in a supine position with a total dose of 0.2 to $0.5 \mathrm{ml} / \mathrm{kg}$ of $0.2 \%$ ropivicaine or $0.25 \%$ bupivacaine with epinephrine 1:200,000 divided in the various interspaces $[5,6]$. There are current clinical trials of wound catheter infusions through $\mathrm{ON}-\mathrm{Q}$ catheters in comparison to IV PCA for postoperative pain control. These wound catheters are inserted by the surgeon just before the closure of the incision. They are connected to an elastomeric pump filled with 0.15 $0.25 \%$ ropivicaine and administered at a constant flow rate of $2 \mathrm{ml} /$ hour in each branch of the catheter for $48 \mathrm{hrs}$.

Perioperative anesthetic and pain management of patients undergoing the Nuss procedure for pectusexcavatum repair is a challenge to practitioners. There are many reviews that have demonstrated that an epidural is effective and safe following pectusexcavatum repair. Besides providing better analgesia, the epidural helps to avoid opioid related adverse effects such as sedation and respiratory depression. In addition, placing thoracic epidural catheters in adolescents when they are awake improves safety against neurological complications. Problems that can be encountered with thoracic epidural analgesia include hypotension, upper extremity weakness or tingling, failed or 
one-sided epidural, Horner syndrome, and in rare but serious cases an epidural hematoma or an epidural abscess. IV PCA in addition to other forms of regional analgesia has also worked equally well at other institutions. Certain analgesic techniques and protocols work well at certain institutions, and there are a variety of outcome measures used within these institutions to help to further improve efficacy and safety.

\section{References}

1. Ravitch MM (1977) Congenital deformities of the chest wall and their operative correction, W.B. Saunders Company, Philadelphia.

2. Nuss D, Kelly RE Jr, Croitoru DP, Katz ME (1998) A 10-year review of a minimally invasive technique for the correction of pectusexcavatum. J PediatrSurg 33(4): 545-552.
3. St Peter SD, Weesner KA, Sharp SW, Sharp RJ, Ostlie DJ, Holcomb GW $3^{\text {rd }}$. J Pediatric Surg 2008 Jan;43(1): 79-82.

4. St Peter SD, Weesner KA, Weissend EE, Sharp SW, Valusek PA, et al. (2012)Epidural vs. patient-controlled analgesia for postoperative pain after pectusexcavatum repair: a prospective, randomized trial. J Pediatr Surg. 47(1): 148-53.

5. Hall-Burton MD, Visoiu MD, Antonio Cassara MD, Katherine Boretsky BS , Franklyn Cladis, et al. (2012) Continuous Paravertebral Nerve Block as an Alternative to Epidural Analgesia for Nuss Procedure. Reg Anesth Pain Med Spring.

6. Bigeleisen P (2009) Ultrasound Guided Regional Anesthesia and Pain Medicine. Springhouse Publishing, Norristown Rd, Philadelphia, pp. 189190. 